\title{
COMENTARIO SOBRE EL METABOLISMO DE METALES PESADOS EN ORGANISMOS MARINOS.
}

por

\section{E.A. Gutiérrez-Galindo}

Instituto de Investigaciones Oceanológicas Apartado Postal 453

Ensenada, Baja California, México.

RESUMEN

Se presenta un breve comentario sobre el metabolismo de metales pesados (en especial cadmio-mercurio) en organismos marinos. Se describen factores biológicos, físicos y físicoquímicos que influyen en la cinêtica de acumulación del elemento por estos organismos.

\section{ABSTRACT}

A brief comment is presented discussing heavy metal metabolism (especially cadmium-mercury) by marine organisms. Biological, physical and physico-chemical factors that influence the kinetics of the element's accumulation by these organisms is described.

\section{E1 mar es considerado actualmente como una solución} potencial del problema alimenticio del mundo, sin embargo, para a.lgunos investigadores, el mar se considera cono el basurero sin fronteras que marca el final de los residuos contaminantes (especialmente los metales pesados) vertidos directa o indirectamente por la acción del hombre. Esta contaminación crónica del medio, puede provocar modificaciones ecológicas susceptibles de amenazar el equilibrio natural y por este hecho, repercutir de manera desfavorable en el nivel de explotación del producto marino alimenticio. La contaminación marina producida por los metales pesados tales como el mercurio y el cadmio, considerados tóxicos para un gran número de organismos marinos, cuando al ser acumulados por estos, constituyen un serio peligro si son destinados a la alimentación humana.

Son conocidas las tragedias ocasionadas por las intoxicaciones producidas por ingestión de peces y moluiscos contaminados por mercurio en los pueblos pesqueros de Minimata y Niigata (Japón) (Tokuomi, 1971). Y la producida por el uso de alimentos y aguas contaminadas por cadmio en determinadas zonas 
del Japón que dieron lugar a la enfermedad conocida por el nombre de "Itai-Itai" (Ay-Ay) (Tsuchiya, 1969; Yamagata y Shigematsu, 1970).

Numerosas industrias utilizan el mercurio. Entre las más importantes se distinguen la industria eléctrica y electrónica (en la fabricación de baterías), la industria farmacéutica (en la fabricación de antisépticos), la industria de la pintura, en los tratamientos de desperdicios nucleares, en la industria de control (en la fabricación de termómetros, barómetros). En lo que se refiere al cadmio, es empleado en la industria metalúrgica, en la industria de colorantes, es incorporado también a ciertos insecticidas y fungicidas.

METABOLISMO Y ACUMULACION DE METALES PESADOS POR LOS ORGANISMOS MARINOS.

\section{Metabolismo}

Algunos metales son oligo-elementos indispensables para organismos y plantas. Sus funciones son diversas: estructurales, catalíticas o electro-químicas. Es admitido que casi todos los elementos menores esenciales poseen una o varias funciones a nivel celular, particularmente enzimática. Numerosas enzimas contienen en efecto, metales pesados, y pueden ser clasificadas en dos grupos: primero, las enzimas que poseen un metal débilmente ligado, el cual en ocasiones puede ser reemplazado por otro; segundo, las metal-enzimas, donde el metal activo está fuertemente ligado (ejemplo, las metal-proteínas). Tal parece que muchos metales están ligados orgánicamente al interior de la célula como un complejo-metal, es el caso del cobalto, el manganeso y el fierro en las plantas (Scott y Ericson, 1955; Bowen, 1966). En cuanto se refiere al mercurio y al cadmio, no existen evidencias de que sean benéficos o biológicamente necesarios para los seres vivos.

\section{Acumulación}

El fenómeno de "concentración biológica de un metal pesado" es el resultado de un proceso biológico (principalmente absorción de metales por los organismos indirectamente a través de la alimentación) o bién de un proceso físico-químico (adsorción directa de los metales a la superficie corporal del orga nismo). Sin embargo, es muy dificil de definir como se efectúa la intoxicación en los organismos, ya que estos dos procesos no son necesariamente alternados.

Varios estudios en cadenas alimenticias marinas han demostrado que las concentraciones de metales pesados más elevadas son casi siempre encontradas en los organismos que ocupan los niveles tróficos inieriores (Aubert, 1972; Aubert et al, 1975). 
Las acumuiaciones de los iones metálicos en los organismos marinos depende de diversos factores:

Biológicos: Anatomía, metabolismo, sexo, edad.

Físicos: Temperatura, oxígeno disuelto, $\mathrm{pH}$.

Físicos-químicos: Naturaleza del estado físico-químico del metal, la concentración del ion en el medio y la salinidad.

La salinidad y la temperatura son factores que influyen notablemente en la toxicidad de los metales pesados en organismos del medio marino. Deben ser considerados con detalle, ya que son factores ecológicos fundamentales para la vida de estos organismos.

En función de la salinidad:

- Diversos metales son más accesibles a la biomasa pelágica en las zonas de aguas con bajas salinidades.

- Las variaciones bruscas de salinidad afectan la tasa de filtración en algunos organismos (especialmente en les moluscos).

- Igualmente, estas variaciones de salinidad dan lugar a ciertos cambios fisiológicos en los organismos, (por ejemplo, el cierre de valvas en los moluscos durante un tiempo prolongado).

En función de la temperatura:

- El cambio brusco de la misma puede activar el metabolismo en los organismos. Generalmente el aumento de la temperatura acelera el ritmo metabólico de estos organismos.

EFECTOS DE METALES PESADOS EN LOS ORGANISMOS MARINOS.

La sensibilidad de los organismos marinos a los metales pesados varía de una especie a ot ta. La previsión de los efectos de dichos contaminantes se calcula a partir de la concentración del elemento químico que ocasiona la muerte da1 $50 \%$ de los organismos er un período de tiempo de cuarenta y ocho o bién de noventa y seis horas. Esta concentración se conoce cono 1a LC 50 (lethal concentration) concentración letal para el $50 \%$ de la población. Ciertos autores prefieren utilizar el TLM (temps letal moyen) tiempo letal promedio para una concentración del elemento que ocasiona la muerte total de la población. 
En cuanto a efecto de metales en organismos, tenemos el caso del cadmio, metal muy tóxico conocido por causar alteraciones en los seres marinos después de una exposición prolongada. Este elemento puede contaminar el aire y la alimentación. Penetra en el organismo por ingestión (Nordberg, 1978). Puede presentar sinergia con otras substancias, aumentando asî su toxicidad (Hublou, 1954). En los peces, el cadmio se acumula principalmente en las branquias, en el riñón y en el hígado; existen evidencias que el buen funcionamiento de dichos ôrganos es afectado (Mount y Stephan, 1967; Gardner y Yevich, 1970). En los organismos marinos (plancton-bivalvos-peces) puede acumularse a concentraciones bastante elevadas $\left(2 \times 10^{6}\right.$ ) (Mullin y Riley. 1956). Este metal inhibe numerosas enzimas, con 10 cual provoca cambios fisiológicos en los organismos (Vallee y Ulmer, 1972).

En 1o que se refiere a otro metal responsable de muchas muertes en Japón, el mercurio, ejerce efectos tales como los señalados por Harris et al (1970). Sus estudios muestran que cantidades de $0.1 \mathrm{ng} / 1$ de metil-mercurio son suficientes para que el mecanismo fotosintético del fitopläncton sea reducido. En los peces, el mercurio se acumula en los tejidos, siendo en el hígado, bazo, riñón e intestino donde generalmente se encuentran las concentraciones más elevadas (Eaton, 1974). En los moluscos (organismos filtradores) las concentraciones de mercurio suelen ser mayores que en los crustáceos (Aubert, 1972). Esto demuestra que los organismos marinos son bastante sensibles a los elementos químicos.

En esta contribución, hemos tratado de describir de una manera general el metabolismo, la cinética de acumulación y los efectos producidos por algunos metales pesados en la naturaleza. Es interesante comenzar a comprender como dichos contaminantes pueden modificar el estado natural del medio acuático. Así mismo, deseamos que esta aportación limitada, active el desarrollo de la investigación de las consecuencias de los diferentes contaminantes presentes en los organismos y en las aguas, y de este modo enriquecer más profundamente el conocimiento de nuestro patrimonio común: e1 mar.

\section{BIBLIOGRAFIA}

AUBI:RT, M., 1972. Pollutions chimiques et chatnes trophodynamiques marines. Rev. Int. Oceanogr. Med., 28, 9-25.

AUPERT, M., R. Bitte1, F. Laumond, M. Barelli, M. Gauthier, B. Donner y M. Romeo, 1975. Utilisation d'une chalne trophodynamique de type benthique pour 1'etude des transferts des polluants métalliques. Rev. Int. Oceanogr. Méd., 39-40, 117-151. 
BOWEN, H.J.M., 1966. Trace elements in biochemistry. Acad. Press Lond. N.Y.

EATON, J.G., 1974. Chronic cadmium toxicity to the bluegil1 (Lepomis machrochirus). Transaction of Amer. Fish. Soc. $103(4): 729-735$.

GARDNER, G., D. Yevich, 1970. Histological and hematological responses of an estuarine teleost to cadmium. J. Fish. Res. Board Canada 27(12):2185-2196.

HARRIS, R., B.D. White y B.R. MacFarlene, 1970. Mercury compounds reduce photosynchesis by plankton. Science, 170, 736 .

HUBLOU, W.F., 1954. The toxicity of zinc or cadmium for chinook salmon. Oreg. Fish. Comm. Res. Briefs, 5.

MOUNT, D.I., C.E. Stephan, 1967. A method of deteciing cadmium poisoning fish. Fish. J. Wildlife Manage, 31:168-172.

MULIN, J.B. y J.P. Riley, 1956. The occurrence of cadmium in sea-water and in marine organisms and sediments. J. Mar. Res. 15(2): 103-122.

NORDBERG, M., 1978. Studies on metallothionein and cadmium. Environ. Res. 15:381-404.

SCOTT, R. y L.E. Ericson, 1955. Some aspects of cobalt metabolism by Rhodymenia palmata with particular reference to vitamin $B_{12}$ content. J. Exp. Bot. 6:348-361.

TOKUOMI, H., 1971. Aspectos médicos de la enfermedad de Minimata. Tribuna Médica, 376:18-19.

TSUCHIYA, K., 1969. Causation of ouch-ouch (itai-itai) disease. Keio J. Med. 18:181-211.

VALLEE, B.L. y P.D. Ulmer, 1972. Biochemical effects of mercury, cadmium and lead. A. Rev. Biochem. 41:91-128.

YAGAMATA, N. e I. Shigematsu, 1970. Cadmium pollution in perspective. Bul1. Inst. Pub1. Health, 19(1):1-27. 